\title{
Planar terahertz metamaterials for strain sensing
}

\author{
Jining Li, ${ }^{* \dagger}$ Charan M. Shah, ${ }^{\ddagger}$ Withawat Withayachumnankul, ${ }^{*}$ Benjamin S. Y. Ung, ${ }^{*}$ \\ Sharath Sriram, ${ }^{\ddagger}$ Madhu Bhaskaran, ${ }^{\ddagger}$ Shengjiang Chang, ${ }^{\dagger}$ and Derek Abbott* \\ ${ }^{*}$ School of Electrical and Electronic Engineering, The University of Adelaide, SA 5005, Australia \\ ${ }^{\dagger}$ Institute of Modern Optics, Nankai University, Tianjin 300071, China \\ ${ }^{\ddagger}$ Functional Materials and Microsystems Research Group, RMIT University, Melbourne, Victoria 3001, Australia \\ Email: andrewli@eleceng.adelaide.edu.au
}

\begin{abstract}
We present two designs of planar terahertz metamaterials for strain sensing. A series of resonance frequency shifts are obtained with differing gap widths when the sensor is under mechanical compression. The subwavelength metamaterial structures exhibit a high-Q resonance resulting in a good sensitivity to mechanical deformation. Because of the penetrability and relatively high spatial resolution of terahertz waves, the proposed metamaterial-based sensors can be embedded in materials for structural sensing.
\end{abstract}

\section{INTRODUCTION AND BACKGROUND}

$\mathbf{M}$ ETAMATERIALS are artificial composites made of periodic subwavelength resonators that can exhibit unusual properties. To date, many different designs of metamaterials have been investigated [1], for example, split ring resonators (SRRs), electric LC resonators, pairs of rods, and fishnets. They have been developed for various applications, for example, superlenses [2], invisibility cloaks [3], perfect absorbers [4], biological sensing [5]. The electromagnetic response of metamaterials can be manipulated via the dimensions, patterns, and compositions of the unit cells. Therefore, effectively, a shift in the resonance frequency can be used to indicate a change in the dimensions of the metamaterial [6]. Metamaterials typically exhibit high-Q resonance, which facilitates accurate detection of any shift in the resonance frequency [7], [8].

Some important features of terahertz metamaterial sensors are non-contact and non-destructive evaluation. As dry, nonpolar and nonmetallic materials are transparent to terahertz radiation, the strain sensor attached to the structure of interest can be read out directly with no requirement of physical connection, allowing the sensor to be concealed by other transparent objects.

In this paper, we consider two sensor designs based on the I-shaped structure or the capped dipole [9]. Instead of rigid substrates typically used in metamaterials, we choose polydimethylsiloxane (PDMS) as a highly flexible substrate. The dielectric constant and loss tangent of PDMS are 2.35 and 0.02-0.04, respectively, across $0.2-2.5 \mathrm{THz}$ band [10]. PDMS has a low surface energy, which allows temporary adhesion to the surface of other materials easily. Based on those aforementioned features, metamaterial-based strain sensors can achieve high sensitivity, miniaturization, and telemetric sensing.

\section{Designs AND Results}

Two terahertz metamaterials are designed for strain sensing. One design is based on an I-shaped structure, suitable for single-axis strain sensing, while the other is a crossed-I structure for dual-axis sensing. The diagrams for the former and latter structures are shown in Fig.1(a) and Fig.2(a), respectively. The physical parameters are as follows $L=60 \mu \mathrm{m}$, $a_{1}=40 \mu \mathrm{m}, a_{2}=30 \mu \mathrm{m}, b=50 \mu \mathrm{m}, w=5 \mu \mathrm{m}$. The metal used in fabrication is gold with $200 \mathrm{~nm}$ thickness, and the substrate is PDMS with $100 \mu \mathrm{m}$ thickness. The terahertz wave propagates along the $z$ axis, with the polarization of the electric field along the central metallic strip.

For the I-shaped design, when the terahertz wave is transmitted through the metamaterial, the incident electric field induces a large accumulation of surface charges in each end of the Istructure. The charges lead to a strong electric field across the gap similar to that in a capacitor. The metallic resonator has a strong dipole resonance at a specific terahertz frequency. The magnitude of the electric field established at the gap depends strongly on the arm length and is inversely proportional to the gap width. A small change in the gap width will strongly affect the resonance frequency. The simulation result shows that the resonance is at $1.00 \mathrm{THz}$, when the gap width is equal to $10 \mu \mathrm{m}$. When a compressive load is applied along the $x$ direction, the deformation mainly occurs in the gap area, causing the gap width to decrease. This increases the gap capacitance, resulting in a red shift in the resonance frequency. The simulation shows that the resonance frequency shifts $10 \mathrm{GHz}$ when the gap size is reduced by $0.7 \mu \mathrm{m}$, and $20 \mathrm{GHz}$ when the gap size is reduced by $1.3 \mu \mathrm{m}$. In the measurement, the sensor is attached to a block of high-density polyethylene (HDPE), onto which the compression is loaded. As shown in Fig.1(b), the measured resonance frequency shifts with the gap width changing under the compression. The resonance frequencies obtained from the simulation and measurement match very well. We believe the small discrepancies are from higher loss in the fabricated PDMS substrate.

Based on the I-shaped sensor introduced above, we developed another sensor that is sensitive to the strain applied in the two orthogonal directions. The detailed structure and dimensions are shown in Fig.2(a). The length of each arm is decreased to $30 \mu \mathrm{m}$. This weakens the electric coupling in the gaps and leads to a slight blue shift in the resonance 
(a)

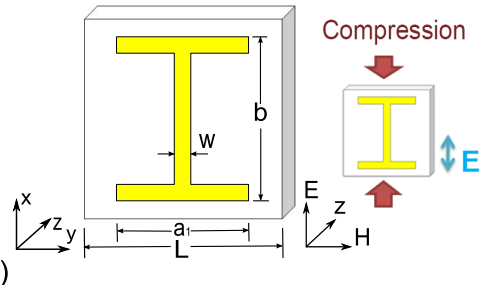

(b)

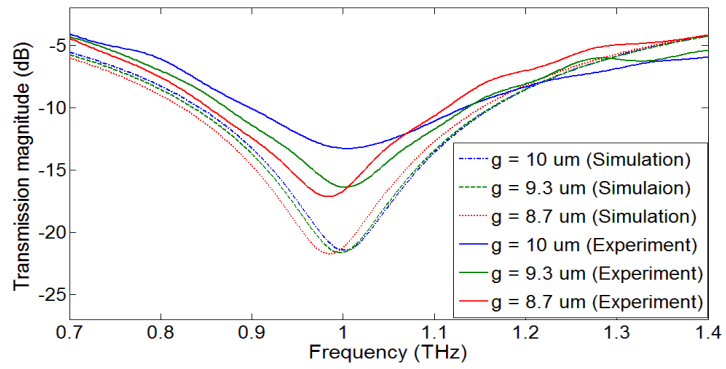

Fig. 1. Single-axis strain sensor. (a) A unit cell of the I-shaped structure with an optical micrograph of the fabricated sensor. (b) The measured and simulated transmission magnitude of I-shaped metamaterial with different gap sizes under applied compression.

frequency [11]. As shown in Fig.2(b), the resonance frequency is $1.15 \mathrm{THz}$ for the gap size of $10 \mu \mathrm{m}$. The frequency shifts approximately $10 \mathrm{GHz}$ and $20 \mathrm{GHz}$ when the gap widths decreases to $9.3 \mu \mathrm{m}$ and $8.7 \mu \mathrm{m}$, respectively. The measurement results prove that the resonance frequency has a red shift with the gap width decreasing under the compression. Because the terahertz wave is linearly polarized, the local electric field is enhanced only in the gaps that are perpendicular to the incident electric-field polarization. In other words, the resonance frequency responds to the deformation only along the polarization direction. An additional experiment shows that the compression deformation from the direction perpendicular to the terahertz wave polarization does not effect the measured resonance frequency, as shown in Fig.3. Therefore, this sensor can be used to sense the strain in the two directions independently.

\section{CONCLUSION}

We present two designs of metamaterial-based terahertz strain sensors. One is suitable for single-axis strain sensing. The other one is designed for dual-axis strain sensing. These sensors are sensitive to mechanical deformation, and are applicable to non-contact and non-destructive evaluation. Future work will involve stretching measurement, and optimization of the sensor designs for a higher sensitivity.

\section{ACKNOWLEDGMENT}

This research was supported by the Australian Research Council Discovery Projects funding scheme under Project DP1095151 (W. Withayachumnankul) and DP1097281 (D. Abbott).

\section{REFERENCES}

[1] W. Withayachumnankul and D. Abbott, "Metamaterials in the terahertz regime," IEEE Photon. J., 1, 2, 99-118, 2009.

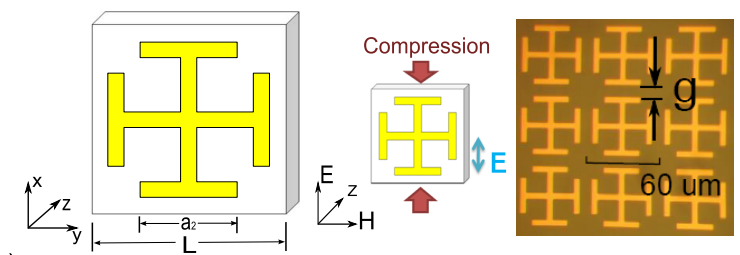

(a)

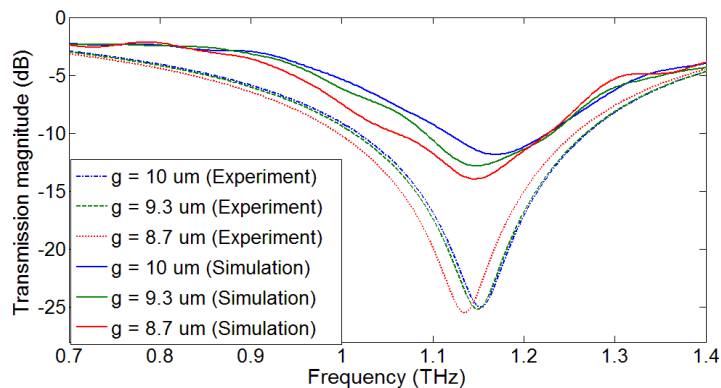

Fig. 2. Dual-axis strain sensor. (a) A unit cell of the crossed-I structure with an optical micrograph of the fabricated sensor. (b) The measured and simulated transmission magnitude of crossed-I metamaterial with different gap sizes under applied compression.

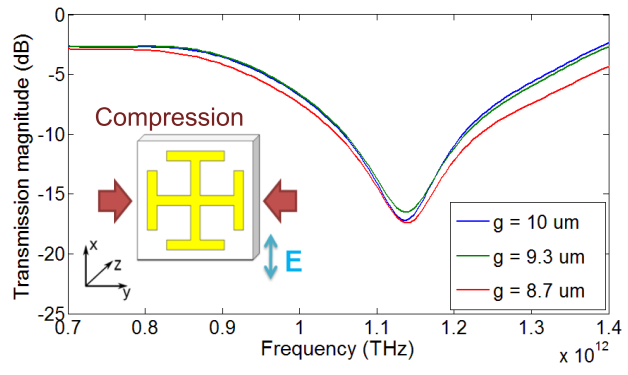

Fig. 3. Measured transmission magnitude of the crossed-I structure as the compression is applied in the direction perpendicular to the electric-field polarization.

[2] D. R. Smith, "How to build a superlens," Science, 308, 502-503, 2005.

[3] W. Cai, U. K. Chettiar, A. V. Kildishev and V. M. Shalaev, "Optical cloaking with metamaterials," Nat. Photonics, 1, 224-227, 2007.

[4] C. M. Watts, X. Liu and W. J. Padilla, "Metamaterial electromagnetic wave absorbers," Adv. Mater., 24, 23, OP98-OP120, 2012.

[5] W. Withayachumnankul, H. Lin, K. Serita, C. M. Shah, S. Sriram, M. Bhaskaran, M. Tonouchi, C. Fumeaux and D. Abbott, "Sub-diffraction thin-film sensing with planar terahertz metamterials," Opt. Express, 20, 3, 3345-3352, 2012

[6] I. M. Pryce, K. Aydin, Y. A. Kelaita, R. M. Briggs and H. A. Atwater, "Highly strained compliant optical matamaterials with large frequency tunability," Nano Lett., 10, 10, 4222-4227, 2010.

[7] R. Melik, E. Unal, N. K. Perkgoz, C. Puttlitz and H. V. Demir, "Metamaterial-based wireless strain sensors," Appl. Phys. Lett., 95, 011106, 2009.

[8] R. Melik, E. Unal, N. K. Perkgoz,B. Santoni, D. Kamstock, C. Puttlitz and H. V. Demir, "Nested metamaterials for wireless strain sensing," IEEE J. Sel. Topics Quantum Electron., 16, 2, 450-458, 2010.

[9] J. Li, W. Withayachumnankul, S. Chang and D. Abbott, "Metamaterialbased strain sensors," in Proc. ISSNIP, 30-32, 2011.

[10] I. E. Khodasevych, C. M. Shah, S. Sriram, M. Bhaskaran, W. Withayachhmnankul, B. S. Y. Ung, H. Lin, W. S. T. Rowe and D. Abbott, "Elastomeric silicone substrates for terahertz fishnet metamaterials," Appl. Phys. Lett., 100, 061101, 2012.

[11] G. Donzelli, A. Vallecchi, F. Capolino and A. Schuchinsky, "Metamaterial made of paired planar conductors: Particle resonances, phenomena and properties," Metamateirals, 3, 1, 10-27, 2009. 